\title{
CARACTERIZAÇÃO CLÍNICA E DAS SITUAÇÕES DE FRATURA DA COLUNA VERTEBRAL NO MUNICÍPIO DE RIBEIRÃO PRETO, PROPOSTAS PARA UM PROGRAMA DE PREVENÇÃO DO TRAUMA RAQUIMEDULAR
}

CLINICAL CHARACTERIZATION AND DESCRIPTION CASES OF VERTEBRAL SPINAL FRACTURE IN THE MUNICIPALITY OF RIBEIRÃO PRETO, PROPOSALS FOR A SPINAL CORD TRAUMA PREVENTION PROGRAM

CARACTERIZACIÓN CLÍNICA Y DE LAS SITUACIONES DE FRACTURA DE LA COLUMNA VERTEBRAL EN EL MUNICIPIO DE RIBEIRÃO PRETO, PROPUESTAS PARA UN PROGRAMA DE PREVENCIÓN DEL TRAUMATISMO RAQUIMEDULAR

Elaine Cristine Lemes Mateus de Vasconcelos ${ }^{1}$, Marcelo Riberto ${ }^{2}$

RESUMO

Objetivo: Este estudo teve como objetivo a caracterização clínica e a avaliação das situações relacionadas ao trauma raquimedular no Hospital das Clínicas da Faculdade de Medicina de Ribeirão Preto durante os anos de 2007 e 2008. Método: Levantamento de prontuários baseado nos códigos da Classificação Internacional de Doenças de fratura da coluna vertebral, exceto aquelas decorrentes de lesão por projétil de arma de fogo. Foram revisados 190 pacientes, 22 tiveram óbito confirmado, 96 foram entrevistados por telefone ou pessoalmente, restando 94 cujas informações foram colhidas apenas a partir do prontuário médico. Resultados: As lesões ocorreram predominantemente nos indivíduos jovens do sexo masculino, acometeram o nível vertebral cervical e causaram lesão medular em 27\% dos indivíduos, principalmente lesões completas (AIS A: 57,7\%). A principal etiologia do trauma foram os acidentes de trânsito com carros e motos (52\%). Nas entrevistas, os pacientes relataram a desatenção e desrespeito às regras de trânsito e normas de segurança como o principal fator associado ao trauma. Muitos pacientes afirmaram desconhecer o risco presente nas situações em que ocorreram os traumas e as consequências da fratura na coluna e lesão medular. Conclusões: Este estudo identificou os homens jovens como sujeitos trauma raquimedular. Entre as situações apontadas pelos pacientes como associadas a esse tipo de trauma, a desatenção e desrespeito às leis foram aspectos importantes. Estas informações permitiram a elaboração de uma campanha de prevenção das lesões da coluna vertebral focada no grupo social e comportamentos de maior risco.

Descritores: Traumatismos da coluna vertebral; Traumatismos da medula espinhal/epidemiologia; Prevenção de acidentes; Fraturas da coluna vertebral.

\begin{abstract}
Objective: This study aimed to characterize clinical aspects and to assess situations related to spinal trauma at the Hospital das Clinicas da Faculdade de Medicina de Ribeirão Preto between 2007 and 2008. Method: we retrieved medical charts International Classification of Diseases codes of spinal fracture, except those caused by gunshots. From 190 patients who were studied, 22 died, 96 were interviewed either by telephone or personally, and 94 had the information collected only from medical charts. Results: fractures occurred mainly in young man, in cervical levels in 27\% of the patients, and were complete in the majority of the cases (AlS A: $57.7 \%$ ). The main etiology of trauma were car and motorcycle accidents (52\%). In the interviews, patients reported lack of attention or disrespect to driving and security rules as the most important factor associated to trauma. Many patients said they did not know the risk in such situations and the consequences of the fractures in the spine or spinal cord injury. Conclusion: this study identified young man as the group most affected by spinal cord injury. Among the most frequent reasons to the trauma according to patients' oppinions were lack of attention or disrespect to security and driving rules. These data permitted the development of a series of preventive actions to spine fractures and spinal cord injuries focused in the specific social group and riskiest behaviors.
\end{abstract}

Keywords: Spinal injuries; Spinal cord injuries/epidemiology; Accident prevention; Spinal fractures.

\section{RESUMEN}

Objetivo: Este estudio tuvo el objetivo de caracterización clínica y evaluación de situações relacionadas con traumatismo raquimedular, en el Hospital de Clínicas de la Facultad de Medicina de Ribeirão Preto, durante 2007-2008. Método: evaluación de historiales de pacientes usando códigos de Clasificación Internacional de Enfermedades relacionados con fractura de columna vertebral, excepto casos

1. Fisioterapeuta do Centro de Reabilitação do Hospital das Clínicas da Faculdade de Medicina de Ribeirão Preto - USP.

2. Professor- Doutor do Departamento de Biomecânica - Medicina e Reabilitação da Faculdade de Medicina de Ribeirão Preto - USP.

Trabalho realizado no Centro de Reabilitação do Hospital das Clínicas da Faculdade de Medicina de Ribeirão Preto da Universidade de São Paulo - São Paulo - Brasil Correspondência: Avenida Bandeirantes, 3900 - Campus Universitário - CEP 14048-900 - Ribeirão Preto - SP - Brasil. 
resultantes de lesión por arma de fuego. Fueron revisados 190 pacientes, 22 tuvieron óbito confirmado, 96 entrevistados por teléfono o personalmente, restando 72 cuyas informaciones fueron recolectadas sólo con el historial médico. Resultados: Las lesiones ocurrieron, predominantemente, en jóvenes varones, acometieron el nivel vertebral cervical y causaron lesión medular en 27\% de los individuos, principalmente lesiones completas (57,7\%). La principal etiología del traumatismo fueron accidentes de tránsito con autos y motos (52\%). En las entrevistas, los pacientes relataron falta de atención, e incumplimiento de reglas de tránsito y normas de seguridad como principales causas del traumatismo. Muchos pacientes afirmaron no conocer el riesgo de las situaciones cuando ocurrieron los traumatismos y las consecuencias de fractura de columna y lesión medular. Conclusiones: Este estudio identificó al grupo de jóvenes como más sujeto a traumatismo con fractura de columna y lesión de médula espinal. Entre los casos señalados por los pacientes como vinculados a este tipo de traumatismo, falta de atención y no cumplimiento de la legislación fueron aspectos importantes. Estos datos permitieron preparar una campaña preventiva de lesiones de columna vertebral, dirigida al grupo social y comportamientos de mayor riesgo.

Descriptores: Traumatismos vertebrales; Traumatismos de la médula espinal/epidemiología; Prevención de accidentes; Fracturas de la columna vertebral.

\section{INTRODUÇÃO}

As fraturas da coluna vertebral são causa importante de morbidade e mortalidade na população mundial, com aumento progressivo nos últimos anos em decorrência da crescente violência urbana, não somente pela sua frequência, mas também pela repercussão funcional e custos individuais e sociais associados às deficiências instaladas ${ }^{1}$. A lesão da medula espinhal ocorre em aproximadamente 15 a $20 \%$ das fraturas da coluna vertebral, com variação entre os diferentes países ${ }^{2}$. No Brasil, estima-se a ocorrência de cerca de 40 novos casos anuais por milhão de habitantes, somando de 6 a 8 mil casos por ano, com custo elevado ao Sistema de Saúde $e^{2,3}$. Nos Estados Unidos, estima-se a ocorrência de 12 mil novos casos de lesão medular ao ano ${ }^{4}$.

A literatura brasileira aponta, como principais causas de lesão medular, os acidentes de trânsito, quedas de altura, mergulhos em água rasa e ferimentos por arma de fogo ${ }^{2}$. A localização anatômica da lesão está diretamente relacionada ao mecanismo de trauma, sendo que a região cervical constitui o segmento mais atingido ${ }^{5}$.

A prevenção deste tipo de trauma exige a identificação das características dos indivíduos envolvidos, causas, níveis de lesão e deficiências, características pessoais e clínicas, além do conhecimento das situações nas quais o mesmo ocorreu, constituindo o ponto de partida para a estruturação de programas preventivos, pois a sua incidência pode variar de acordo com o local e o momento histórico 6 .

O objetivo deste estudo foi a obtenção de informações epidemiológicas sobre a população afetada e as causas mais frequente de fraturas da coluna vertebral atendidos em nosso local de trabaIho. O relato pessoal dos pacientes a respeito do contexto em que ocorreram as fraturas e qual seria a abordagem preventiva segundo a opinião deles complementaram o objetivo do estudo.

\section{MÉTODO}

Foram coletados dados retrospectivos de 202 prontuários médicos de pacientes atendidos no Hospital das Clínicas da Faculdade de Medicina de Ribeirão Preto da Universidade de São Paulo (HCFMRP-USP), no período de 2007 e 2008. Os prontuários foram localizados a partir dos códigos da Classificação Internacional de Doenças (CID) correspondentes à fratura dos vários segmentos da coluna vertebral, além de luxação e traumatismo envolvendo a medula espinhal. Não foram incluídos os casos nos quais a lesão foi causada por projétil de arma de fogo.

Foram excluídos da pesquisa 12 prontuários cujo preenchimento da CID estava incorreto. Além das informações obtidas do prontuário, também foram realizadas entrevistas por telefone com os indivíduos que puderam comparecer ao local da realização do estudo (70). Quando o contato telefônico não foi possível, foi enviada correspondência para o endereço cadastrado junto ao hospital, convidando os pacientes a entrar em contato com os pesquisadores (26).
Foram sistematicamente colhidas informações pessoais como: sexo, idade, mês de ocorrência da fratura, nível da fratura da coluna vertebral, mecanismo de trauma e quadro clínico-funcional, utilizando ficha de coleta estruturada desses dados. Durante os contatos telefônicos e pessoais, foram realizadas entrevistas semi-estruturadas para a coleta de informações sobre o local do acidente e uso ou não de equipamentos de proteção individual (EPI) no local de trabalho, bem como o questionamento direto sobre as circunstâncias que provocaram o trauma e os possíveis aspectos preventivos envolvidos.

\section{RESULTADOS}

Foram verificados 22 óbitos entre os 190 indivíduos identificados. Dos 168 pacientes que restaram, foi efetuada a entrevista por telefone com 70 pacientes. Dos restantes, apenas 26 entraram em contato para a realização da entrevista após contato por correio. Portanto, a análise dos resultados incluiu:

- 96 (50,5\%) pacientes: submetidos à entrevista acrescida de análise do prontuário médico;

- 94 (49,5\%) pacientes, incluindo os óbitos: dados obtidos apenas da análise do prontuário médico.

A média de idade foi 38 17 anos, e 156 (82\%) dos pacientes eram do sexo masculino.

A ocorrência do trauma foi homogênea ao longo do ano, variando de 12 registros de casos nos meses de janeiro e maio, a 20 registro de casos no mês de junho (Figura 1).

Na avaliação do segmento vertebral, a coluna cervical foi a mais acometida, correspondendo a 45\% dos casos analisados (Figura 2).

Dos 190 pacientes estudados, 52 (27\%) apresentaram lesão neurológica, sendo paraplegia completa (16 pacientes - 30,8\%), tetraplegia completa (14 pacientes - 26,9\%), lesão medular cervical incompleta ( 11 pacientes - 21,2\%), lesão medular torácica ou

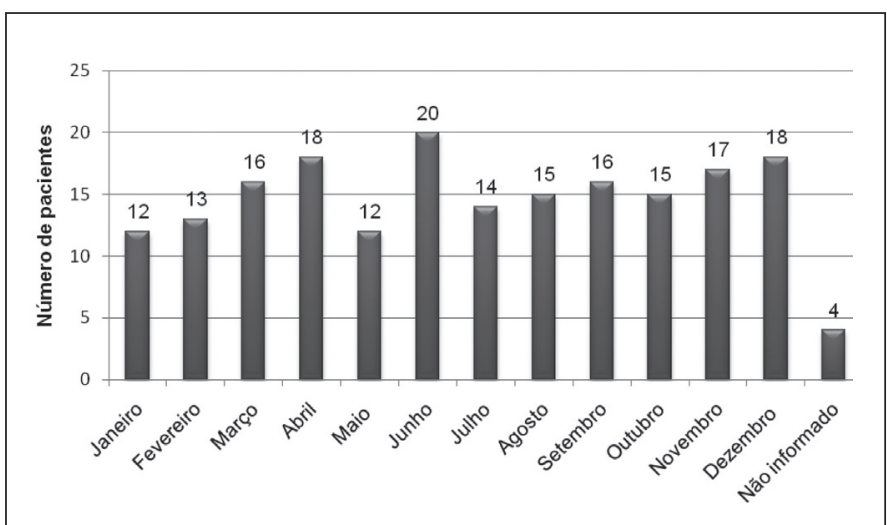

Figura 1. Ocorrência mensal de fraturas da coluna vertebral atendidas no Hospital das Clínicas da Faculdade de Medicina de Ribeirão Preto-USP. 


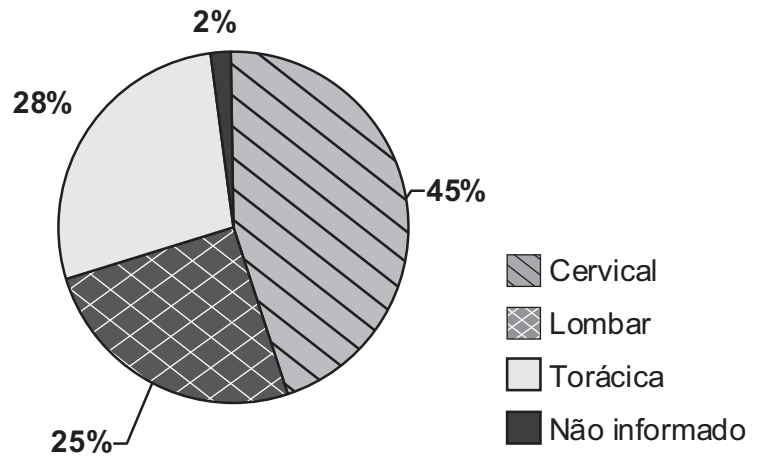

Figura 2. Distribuição dos pacientes de acordo com a localização da fratura nos segmentos da coluna vertebral.

lombar incompleta (6 pacientes - 11,5\%), e outras deficiências (5 pacientes-9,6\%). Cento e trinta e oito pacientes (73\%) não tiveram deficiência neurológica.

Quanto à causa do trauma, houve predomínio de acidentes de trânsito (100 pacientes - 52\%), seguido de quedas (68 pacientes - 36\%) e mergulho (10 pacientes - 5\%), como detalhado abaixo:

- Acidente de trânsito: correspondeu a 52\% (100 pacientes) da amostra, sendo que o veículo da vítima na ocasião do trauma foi motocicleta em 50\% dos pacientes, carro em 29\%, e outros (caminhão, ônibus, bicicleta, atropelamento) em 21\%. Entre os 33 usuários de carro, caminhão e ônibus que deveriam estar usando cinto de segurança no momento do acidente, oito o faziam, sete não estavam utilizando, e em 18 não foi obtida informação.

Entre os 54 usuários de moto e bicicleta que deveriam estar utilizando capacete, 29 confirmaram a sua utilização, e oito não. A informação não foi obtida em 17 pacientes. Merece destaque que dentre os 29 indivíduos que estavam utilizando capacete, oito (27\%) descreveram o uso desse equipamento de forma incorreta.

Dos 67 pacientes que responderam o questionamento quanto às infrações de trânsito e condições das vias públicas que favoreceram o acidente, 23 relataram desrespeito às leis de trânsito e 17 referiram a necessidade de mais sinalização, entre outros fatores, como demonstrado na Figura 3. Cinco pacientes entrevistados deram duas respostas.

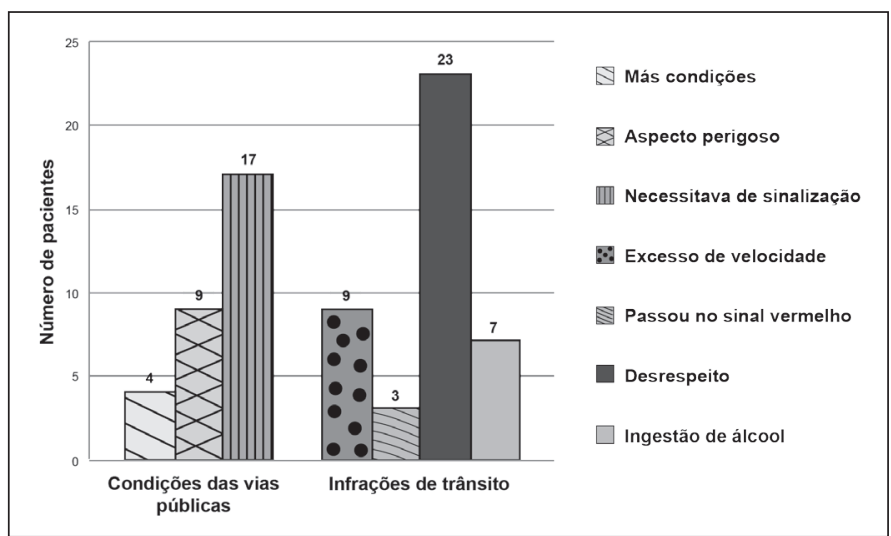

Figura 3. Condições e infrações de trânsito, conforme relato dos pacientes entrevistados.

- Queda: A queda do telhado e laje destacou-se em relação aos demais tipos, correspondendo a 18 (26\%) pacientes entre 68 ocorrências. O restante está distribuído em queda da árvore, cama, escada, rede, entre outras.
- Mergulho: O mergulho na piscina predominou (cinco pacientes), seguido do mergulho em lago (dois pacientes), rio (2 pacientes) e praia (um pacientes).

\section{ENTREVISTAS}

Na realização das 96 entrevistas, foi possível evidenciar acidentes de trabalho em 42 pacientes, enquanto que em 44 pacientes o episódio ocorreu durante atividade habitual ou de lazer, incluindo comemoração/festas, passeio, férias, esporte e serviços domésticos. Em 10 pacientes não foi obtida a informação.

Durante o discurso livre dos pacientes sobre as circunstâncias que favoreceram o trauma, enumeraram-se a imprudência (30 relatos) e a falta de atenção (25 relatos), seguidas de fatalidade (11 relatos) e abuso de álcool (5 relatos). Outros fatores mencionados foram excesso de velocidade, falta de sinalização, desconhecimento do risco, EPI em condições precárias e não utilização de EPI.

Um aspecto a ser salientado refere-se ao desconhecimento por parte de 51 (53,1\%) pacientes entrevistados quanto à gravidade da lesão da coluna vertebral. Apenas 28 pacientes relataram o conhecimento da gravidade e 17 não souberam informar.

Dos 86 pacientes que responderam o questionamento sobre os aspectos preventivos do trauma da coluna vertebral, a necessidade de mais atenção, prudência e cautela (20 pacientes), o uso adequado de EPI (18 pacientes) e o respeito às leis de trânsito (13 pacientes) foram os mais citados. Outros aspectos relacionados à prevenção foram o reforço da sinalização, a fiscalização, a criação de campanhas educativas, a contração de profissional habilitado para a realização de tarefas específicas (principalmente envolvendo atividades no telhado), e a manutenção adequada do veículo de transporte. Apenas um paciente mencionou que não haveria como prevenir o trauma da coluna vertebral.

\section{DISCUSSÃO}

Neste estudo foi observada maior prevalência de fraturas da coluna vertebral nos indivíduos do sexo masculino (82\%) com média de idade de $38 \pm 17$ anos, o que é concordante com outros estudos nacionais ${ }^{7-9}$, e é habitualmente explicado pelo fato de os homens jovens se exporem mais às atividades de risco e possuírem comportamento mais desafiador.

Segundo Silva e Defino ${ }^{10}$, existe sazonalidade na ocorrência da lesão traumática da medula espinhal ocasionada por mergulho em água rasa, já que o maior número dessas lesões ocorre nos meses em que a temperatura está mais elevada. A sazonalidade das lesões traumáticas da coluna vertebral não foi observada em nosso estudo, e pode estar relacionada com a inclusão de diferentes etiologias da lesão traumática, mas demonstra que a sua ocorrência ocorre durante todo o ano.

$\mathrm{Na}$ análise do segmento vertebral mais acometido, a coluna cervical predominou com $45 \%$ dos pacientes estudados, seguida de $28 \%$ de traumas no segmento torácico e $25 \%$ no segmento lombar. Mello et al. ${ }^{11}$ também observarm que a coluna cervical foi a mais acometida, totalizando $37 \%$ das fraturas, seguida pela transição toracolombar. Em contrapartida, segundo Zaninelli et al. ${ }^{12}$, a região compreendida entre T12 e L2 é a sede de mais de 50\% de todas as fraturas da coluna vertebral. Tuono ${ }^{1}$, ao analisar as internações hospitalares decorrentes de traumas de coluna no Brasil, no ano de 2000 a 2005, verificou que, segundo o nível vertebral acometido, houve predomínio das internações por trauma lombo-sacral, seguido por trauma cervical e torácico. Por outro lado, ao analisar o comprometimento da medula segundo o nível da lesão, o nível cervical foi o mais afetado, em todos os anos.

Trinta pacientes (15\%) apresentaram lesão medular completa, sendo 16 (8\%) paraplegia e 14 (7\%) tetraplegia. Dezessete pacientes (9\%) tiveram lesão medular incompleta e 138 (73\%) não tiveram deficiência, seguindo as proporções observadas por Mello et al. ${ }^{11}$. 
Todavia, dados norte-americanos sobre o nível neurológico e a extensão da lesão mostram que os padrões de comprometimento mais frequentes são tetraplegia incompleta $(38,3 \%)$, seguida por paraplegia completa $(22,9 \%)$, paraplegia incompleta $(21,5 \%)$ e tetraplegia completa (16,9\%), com o aumento percentual de tetraplegia incompleta e redução de lesões completas ${ }^{4}$, o que está associado à qualidade do atendimento pré-hospitalar.

Outro aspecto estudado foi a etiologia do trauma e as circunstâncias da sua ocorrência. Observamos como mecanismos de trauma mais prevalentes, os acidentes de trânsito, quedas de altura e acidente por mergulho. Esses achados são relatados em outros estudos nacionais ${ }^{2,13}$. Silva e Defino ${ }^{10}$ realizaram estudo epidemiológico das fraturas da coluna cervical nos pacientes atendidos nos hospitais da cidade de Ribeirão Preto-SP, no período de janeiro de 1989 a dezembro de 1999, e verificaram igualmente que os acidentes automobilísticos se sobressaem, seguidos de queda de altura e mergulho em água rasa, o que vai ao encontro dos estudos internacionais ${ }^{4,14}$

Em contrapartida, Tuono ${ }^{1}$ verificou que, quanto às causas externas que ocasionaram os traumas de coluna no ano de 2000 a 2005, as quedas corresponderam a cerca de $40 \%$ do total de internações em todos os anos estudados, seguidas por acidentes de transporte com frequência de 22,7\% em 2005. Outros acidentes, que incluem o mergulho em águas rasas, foram responsáveis, em 2005, por quase $20 \%$ dos casos. Semelhantemente, outros autores $8,9,11,12,15,16$ verificaram que os mecanismos de trauma mais prevalentes foram queda, seguidos de acidentes de trânsito, dados não encontrados no nosso estudo, talvez porque a maior parte das pesquisas aborda regiões ou locais específicos, gerando essa variabilidade quanto à etiologia.

A maior parte dos pacientes entrevistados relatou desrespeito às leis de trânsito, seguido pela necessidade de melhor sinalização das vias públicas, o que foi reforçado pela evidência de não terem usado cinto de segurança ou capacete na ocasião do acidente, além do uso inadequado do capacete. Estes achados mostram a necessidade de se investir ainda mais na conscientização quanto à importância de se respeitar as leis de trânsito, incluindo a obrigatoriedade do uso desses equipamentos.

Os entrevistados ainda citaram a imprudência e a falta de atenção como circunstâncias que favoreceram o trauma. Somadas a elas estavam: EPI em condições precárias ou não utilização de EPI, e desconhecimento do risco. O fato da pessoa não ter a percepção de que está em risco foi relatada por Silva e Defino ${ }^{10}$ ao entrevistar pacientes tetraplégicos vítimas do mergulho em águas rasas. Eles verificaram que todos desconheciam o tipo de acidente e suas consequências, e que a preocupação que tinham em relação ao lazer aquático estava voltada ao afogamento e não à possibilidade de fratura da coluna cervical.

\section{REFERÊNCIAS}

1. Tuono VL. Traumas de coluna no Brasil: análise das internações hospitalares (dissertação). Ribeirão Preto: Faculdade de Saúde Pública, Universidade de São Paulo; 2008.

2. Defino HLA. Trauma raquimedular. Medicina, Ribeirão Preto. 1999:32(11/12):388-400

3. Greve JM. Traumatismos raquimedulares nos acidentes de trânsito e uso de equipamentos de segurança. Diagn \& Trat. 1997;2(1):10-3.

4. National Spinal Cord Injury Statistical Center. Spinal cord injury facts and figures at a glance. J Spinal Cord Med. 2010;33(4):439-40.

5. Slucky AV, Eismont FJ. Treatment of acute injury of the cervical spine. J Bone Joint Surg Am. 1994;76:1882-9

6. Gonçalves AMT, Rosa LN, D'Ângelo CT, Savordelli CL, Bonin GL, Squarcino IM, Borrelli M. Aspectos epidemiológicos da lesão medular traumática na área de referência do Hospital Estadual Mário Covas. Arq Med ABC. 2007;32(2):64-6.

7. Bambi LNS, Guilhem D, Lima DD. Qualidade de vida em pessoas com lesão medular traumática: um estudo com o WHOQOL-bref. Rev Bras Epidemiol. 2008;11(1):67-77.

8. Campos MF, Ribeiro AT, Listik S, Pereira CAB, Andrade Sobrinho J, Rapoport A. Epidemiologia do traumatismo da coluna vertebral. Rev Col Bras Cir. 2008;35(2):88-93.

9. Oliveira PAS, Pires JV, Borges Filho JMM. Traumatismos da coluna torácica e lombar. Avaliação epidemiológica. Rev Bras Ortop. 1996;31(9):771-6.
Por outro lado, devemos salientar que, segundo a afirmação espontânea de alguns pacientes do nosso estudo, eles acreditavam que estavam isentos da possibilidade de sofrer um acidente, negligenciando o risco. Acrescido a isso, as entrevistas revelaram que grande parte das pessoas desconhece a gravidade de se lesar a coluna vertebral e a medula espinhal.

Constatamos ainda que, na ocasião do trauma envolvendo a coluna vertebral, não houve uma discrepância quanto à caracterização em acidente de trabalho ou acidente decorrente de atividade habitual ou de lazer (comemoração/ festas, passeio, férias, esporte e serviços domésticos), apontando que as campanhas de prevenção devem abranger ambas as situações.

Quanto ao aspecto preventivo relacionado ao trauma de coluna, a maior parte dos pacientes entrevistados relatou a necessidade de mais atenção, prudência e cautela. Outros fatores mencionados foram a importância de respeitar as leis de trânsito e usar adequadamente os EPI. Esses achados têm relação com as circunstâncias citadas pelos pacientes que favoreceram a ocorrência do acidente. Alguns pacientes reforçaram a necessidade de se criar campanhas preventivas e contratar profissional habilitado para realizar um determinado trabalho, especialmente no telhado, evitando o risco de queda. Somente um paciente relatou que não é possível prevenir o trauma de coluna.

Os dados obtidos com o presente estudo nos motivaram e embasaram a elaboração de uma campanha educativa com ações voltadas à prevenção do trauma da coluna vertebral (www.colunafragil. org.br). Vale ressaltar que o relato pessoal dos pacientes enriqueceu ainda mais a pesquisa, permitindo criar o conceito da campanha.

\section{CONCLUSÃO}

As informações epidemiológicas obtidas quanto às fraturas da coluna vertebral e lesão medular mostraram que os principais acometidos são homens jovens, em situação de trânsito (carro ou motocicleta). Não houve variação sazonal na incidência, e na maior parte das vezes não houve lesão nervosa. Quando a lesão medular ocorreu, a principal manifestação foi a paraplegia completa.

$O$ relato pessoal dos pacientes entrevistados permitiu concluir que, quanto aos acidentes de trânsito, o desrespeito às leis de trânsito foi referido como a principal causa, associado principalmente à desatenção e desinformação quanto à possibilidade de lesões em várias situações corriqueiras e a respeito da gravidade das conseqüências do trauma raquimedular.

O estudo permitiu elaborar material para uma campanha de prevenção do trauma raquimedular, alertando para situações de maior risco e a necessidade de atenção às medidas habituais de prevenção de acidentes no trânsito, trabalho e lazer, além de identificar grupos mais suscetíveis ao trauma que merecem ações mais enfáticas.

10. Silva CLC, Defino HLA. Estudo epidemiológico das fraturas da coluna cervical por merguIho na cidade de Ribeirão Preto-SP. Medicina (Ribeirão Preto). 2002;35(1):41-7.

11. Mello LR, Espíndola G, Silva FM, Bernardes Cl. Lesado medular. Estudo prospectivo de 92 casos. Arq Bras Neurocir. 2004;23(4):151-6.

12. Zaninelli EM, Graells XSI, Néri OJ, Dau L. Avaliação epidemiológica das fraturas da coluna torácica e lombar de pacientes atendidos no Pronto-Socorro do Hospital do Trabalhador da UFPR de Curitiba - Paraná. Coluna/Columna. 2005;4(1):11-5.

13. Tavares JP, Leal AIC, Paiva WS, Masini M. Estudo descritivo da lesão medular em unidades de neurocirurgia de 80 hospitais gerais brasileiros. Acta Cir Bras. 2001;16 (Suppl 1):134-6.

14. Jackson AB, Dijkers M, DeVivo MJ, Paczatek RB. A demographic profile of new traumatic spinal cord injuries: change and stability over 30 years. Arch Phys Med Rehabil. 2004;85(11):1740-8.

15. Koch A, Graelss XSI, Zaninelli EM. Epidemiologia de fraturas da coluna de acordo com o mecanismo de trauma: análise de 502 casos. Coluna/Columna. 2007:6(1):18-23.

16. Siscão MP, Pereira C, Arnal RLC, Foss MHD, Marino LHC. Trauma raquimedular: caracterização em um hospital público. Arq Cienc Saúde. 2007;14(3):145-7. 